\title{
ESTIMATIONS DES SOLUTIONS DE L'ÉQUATION DE BEZOUT DANS LES ALGĖBRES DE BEURLING ANALYTIQUES
}

\author{
O. EL-FALLAH et M. ZARRABI
}

\begin{abstract}
Let $A$ be a unitary commutative Banach algebra with unit $e$. For $f \in A$ we denote by $\hat{f}$ the Gelfand transform of $f$ defined on $\hat{A}$, the set of maximal ideals of $A$. Let $\left(f_{1}, \ldots, f_{n}\right) \in A^{n}$ be such that $\sum_{i=1}^{n}\left\|f_{i}\right\|^{2} \leq 1$. We study here the existence of solutions $\left(g_{1}, \ldots, g_{n}\right) \in A^{n}$ to the Bezout equation $f_{1} g_{1}+\cdots+f_{n} g_{n}=e$, whose norm is controlled by a function of $n$ and $\delta=\inf _{\chi \in \hat{A}}\left(\left|\hat{f}_{1}(\chi)\right|^{2}+\cdots+\left|\hat{f}_{n}(\chi)\right|^{2}\right)^{1 / 2}$.

We treat this problem for the analytic Beurling algebras and their quotient by closed ideals. The general Banach algebras with compact Gelfand transform are also considered.
\end{abstract}

\section{Introduction}

Soit $A$ une algèbre de Banach commutative unitaire. L'ensemble des caractères de $A$, qu'on appellera aussi spectre de $A$, sera noté $\hat{A}$. La transformée de Gelfand associée à $A$ est l'application:

$$
\begin{aligned}
\mathscr{G}: A & \longrightarrow \mathscr{C}(\hat{A}) \\
f & \longrightarrow \hat{f}
\end{aligned}
$$

où

$$
\hat{f}(\chi)=\chi(f), \quad \chi \in \hat{A},
$$

et où $\mathscr{C}(X)$ désigne l'ensemble des fonctions continues sur l'espace topologique $X$.

Rappelons maintenant les différentes versions quantitatives de la visibilité du spectre introduites dans [5] et [9]. Pour tout entier $n \geq 1$ et tout $\delta \in(0,1]$, on dit que le spectre de $A$ est $(\delta-n)$-visible ([5]) s'il existe une constante $C \in(0,+\infty)$ tel que pour tout $f=\left(f_{1}, f_{2}, \ldots, f_{n}\right) \in A^{n}$ satisfaisant

(1) $\sum_{i=1}^{n}\left\|f_{i}\right\|^{2} \leq 1 \quad$ et $\quad \sum_{i=1}^{n}\left|\hat{f}_{i}(\chi)\right|^{2} \geq \delta^{2}$ pour tout $\chi \in \hat{A}$,

Received January 25, 2004. 
il existe $g=\left(g_{1}, g_{2}, \ldots, g_{n}\right) \in A^{n}$ tel que

$$
\sum_{i=1}^{n} f_{i} g_{i}=e \quad \text { et } \quad\left(\sum_{i=1}^{n}\left\|g_{i}\right\|^{2}\right)^{\frac{1}{2}} \leq C .
$$

où $e$ est l'élément unité de $A$.

On notera alors

$$
C_{n}(\delta, A)=\sup \left\{\inf \left\{\left(\sum_{i=1}^{n}\left\|g_{i}\right\|^{2}\right)^{\frac{1}{2}}: \sum_{i=1}^{n} f_{i} g_{i}=e\right\}\right\},
$$

le sup étant pris sur les éléments $f$ de $A^{n}$ satisfaisant (1). Notons que le spectre de $A$ est $(\delta-n)$-visible pour $\delta \in] 0,1]$ si et seulement si $C_{n}(\delta, A)<+\infty$. Il est clair que si $0<\delta^{\prime} \leq \delta \leq 1$, alors $C_{n}(\delta, A) \leq C_{n}\left(\delta^{\prime}, A\right)$. Donc il existe un point critique $\delta_{n}(A) \in[0,1]$ tel que $C_{n}(\delta, A)=+\infty$ pour $\delta<\delta_{n}(A)$ et $C_{n}(\delta, A)<+\infty$ pour $\delta>\delta_{n}(A)$. Signalons aussi que $C_{n}(\delta, A) \leq C_{n+1}(\delta, A)$ pour tout $n \geq 1$.

On dit que le spectre de $A$ est $\delta$-Complétement visible s'il est $(\delta-n)$-visible pour tout $n \geq 1$ et $\sin _{\sup _{n \geq 1}} C_{n}(\delta, A)<+\infty$.

On notera dans la suite, $\mathrm{T}$ le cercle unité, $\mathrm{D}$ le disque unité, pour tout $f \in \mathscr{C}(\mathrm{T}), \hat{f}(n)=\frac{1}{2 \pi} \int_{0}^{2 \pi} f\left(e^{i t}\right) e^{-i n t} d t$ le $n^{\text {ième }}$ coefficient de Fourier de $f$ et enfin $A(\mathrm{D})$ l'algèbre des fonctions continues sur $\overline{\mathrm{D}}$ et holomorphe dans $\mathrm{D}$.

Dans ce travail $\omega$ désignera une fonction croissante de $\mathrm{N}$ à valeurs dans ] $0,+\infty[$ vérifiant

$$
\omega(0)=1, \quad \omega(n+m) \leq \omega(n) \omega(m), \quad(n, m \in \mathrm{N}) \text { et } \lim _{n \rightarrow+\infty} \omega(n)^{\frac{1}{n}}=1 .
$$

L'algèbre de Beurling analytique associée au poids $\omega$ est l'espace

$$
A_{\omega}^{+}=\left\{f \in A(\mathrm{D}): \sum_{n \in \mathrm{N}}|\hat{f}(n)| \omega(n)<+\infty\right\},
$$

muni du produit ponctuel et de la norme $\|f\|_{\omega}=\sum_{n \in \mathrm{N}}|\hat{f}(n)| \omega(n)$. Ainsi $A_{\omega}^{+}$ est une algèbre de Banach commutative unitaire semi-simple. En identifiant son spectre au disque unité fermé, la transformée de Gelfand devient l'identité i.e $\hat{f}=f$ pour tout $f \in A_{\omega}^{+}$. Notons que $A_{\omega}^{+}$est isomorphe à l'algèbre de Beurling sur les entiers naturels $\ell_{\omega}^{1}=\left\{\left(x_{n}\right)_{n \geq 0}: \sum_{n \in \mathrm{N}}\left|x_{n}\right| \omega(n)<+\infty\right\}$.

La caractérisation des algèbres de Beurling-Sobolev ayant la $(\delta-1)$-visibilité a fait l'objet de plusieurs travaux (voir [5], [9], [4], [10] et [1]) . Il a été démontré dans [4], que $\delta_{1}\left(A_{\omega}^{+}\right)=0$ si et seulement si

$$
\lim _{p \rightarrow+\infty} a(p, \omega)=0
$$


où

$$
a(p, \omega)=\lim _{q \rightarrow+\infty} a(q, p, \omega)
$$

et

$$
a(q, p, \omega)=\sup \left\{\left(\frac{\omega\left(n_{1}+n_{2}+\cdots+n_{p}\right)}{\omega\left(n_{1}\right) \omega\left(n_{2}\right) \ldots \omega\left(n_{p}\right)}\right)^{\frac{1}{p}}: n_{i} \geq q(i=1,2, \ldots, p)\right\} .
$$

Notons que toute suite croissante $\omega$ qui satisfait la condition (4) diverge nécessairement vers l'infini.

Notre objectif dans ce travail est d'étudier la $(\delta-n)$-visibilité pour les algèbres de Beurling analytiques et pour les quotients de ces algèbres. Les deux résultats principaux de ce travail sont:

ThÉORÈme A. Soit $\omega$ une suite croissante satisfaisant (3). Alors les assertions suivantes sont équivalentes.

i) Le spectre de $A_{\omega}^{+}$est $\delta$-complétement visible pour tout $\delta \in(0,1]$.

ii) Pour tout $n \geq 1$, le spectre de $A_{\omega}^{+}$est $(\delta-n)$-visible pour tout $\delta \in(0,1]$.

iii) w satisfait la condition (4).

THÉORÈME B. Soit $\omega$ une suite croissante satisfaisant (3). Alors les assertions suivantes sont équivalentes.

i) w satisfait la condition (4).

ii) Pour tout idéal fermé I de $A_{\omega}^{+}$et pour tout $\delta \in(0,1]$, le spectre de $A_{\omega}^{+} / I$ est $\delta$-complétement visible.

Les théorèmes $\mathrm{A}$ et $\mathrm{B}$ sont démontrés respectivement dans la section 2 et la section 3. Dans la section 4 nous considérons plus généralement les algèbres dont la transformée de Gelfand est compacte.

\section{Preuve du Théorème $A$}

Soit $\omega$ une suite croissante satisfaisant (3) et soit $\widetilde{\omega}$ le poids symétrique sur Z associé à $\omega$ (i.e $\widetilde{\omega}(n)=\omega(|n|)$ pour tout $n \in \mathbf{Z})$. L'espace

$$
A_{\widetilde{\omega}}=\left\{f \in \mathscr{C}(\mathbf{T}): \sum_{n \in Z}|\hat{f}(n)| \widetilde{\omega}(n)<+\infty\right\},
$$

muni du produit ponctuel et de la norme $\|f\|_{\widetilde{\omega}}:=\sum_{n \in Z}|\hat{f}(n)| \widetilde{\omega}(n)$ est une algèbre de Banach commutative unitaire dont l'ensemble des caractères s'identifie au cercle unité $\mathrm{T}$. Lorsque $\widetilde{\omega}(n)=1$ pour tout $n \in \mathbf{Z}$, on écrira $\|f\|_{1}$ au lieu de $\|f\|_{\widetilde{\omega}}$. Puisque le poids $\omega$ est croissant on a $\delta_{1}\left(A_{\omega}^{+}\right)=0$ si et seulement si $\delta_{1}\left(A_{\widetilde{\omega}}\right)=0$ ([4], Corollaire 5.5). 
Dans le Théorème $\mathrm{A}$, l'implication i) $\Rightarrow$ ii) est triviale, l'implication ii) $\Rightarrow$ iii) découle immédiatement de [4] et enfin l'implication iii) $\Rightarrow$ i) est une conséquence directe de [4] et du résultat suivant.

ThÉORÈme 2.1. Soit $\omega$ une suite croissante satisfaisant (3). Pour tout $\delta \in$ $(0,1]$ et tout $n \geq 1$ on a

$$
C_{n}\left(\delta, A_{\omega}^{+}\right) \leq 5 C_{1}\left(\delta^{2}, A_{\widetilde{\omega}}\right)^{2} .
$$

Preuve. Soient $\delta \in(0,1]$ et $f=\left(f_{1}, \ldots, f_{n}\right) \in\left(A_{\omega}^{+}\right)^{n}$ satisfaisant

$$
\sum_{i=1}^{n}\left\|f_{i}\right\|_{\omega}^{2} \leq 1 \quad \text { et } \quad \sum_{i=1}^{n}\left|f_{i}(\zeta)\right|^{2} \geq \delta^{2} \quad \text { pour tout } \zeta \in \overline{\mathrm{D}} .
$$

Nous allons construire $g=\left(g_{1}, \ldots, g_{n}\right) \in\left(A_{\omega}^{+}\right)^{n}$ satisfaisant

$$
\sum_{i=1}^{n} f_{i} g_{i}=1 \quad \text { et } \quad \sum_{i=1}^{n}\left\|g_{i}\right\|_{\omega}^{2} \leq 25 C_{1}\left(\delta^{2}, A_{\widetilde{\omega}}\right)^{4} .
$$

Dans un premier temps nous supposons que les $f_{j}, j=1,2, \ldots, n$, sont holomorphes au voisinage de $\overline{\mathrm{D}}$. Soient $F=\sum_{j=1}^{n}\left|f_{j}\right|^{2}$ et $h_{j}=\frac{\overline{f_{j}}}{F}$. Il est clair que les restrictions de $F$ et $h_{j}$ au cercle unité appartiennent à $A_{\widetilde{\omega}}$ et que $\sum_{j=1}^{n} f_{j} h_{j}=1$. Comme dans la preuve du théorème de la couronne dans $H^{\infty}$, nous allons modifier les $h_{j}$ pour obtenir des solutions holomorphes sur D. Pour cela soit $a_{j, k}=h_{j} \frac{\partial h_{k}}{\partial \bar{z}}-h_{k} \frac{\partial h_{j}}{\partial \bar{z}}$ et

$$
b_{j, k}(z)=-\frac{1}{\pi} \int_{\mathrm{D}} \frac{a_{j, k}(\zeta)}{\zeta-z} d A(\zeta),
$$

où $d A$ est la mesure d'aire. Puisque les $h_{j}, j=1,2, \ldots, n$, sont $C^{\infty}$ dans un voisinage de $\overline{\mathrm{D}}$, il est bien connu que $b_{j, k}$ l'est aussi et que $\frac{\partial b_{j, k}}{\partial \bar{z}}=a_{j, k}$ sur D. Soit

$$
g_{j}=h_{j}+\sum_{k=1}^{n} b_{j, k} f_{k} .
$$

Il est alors clair que $g_{j}$ est holomorphe sur D et que $\sum_{j=1}^{n} f_{j} g_{j}=1$. Le reste de la preuve sera consacrée à la majoration de $\sum_{j=1}^{n}\left\|g_{j}\right\|_{\omega}^{2}$.

Nous avons

$$
\left\|g_{j}\right\|_{\omega}=\left\|h_{j}+\sum_{k=1}^{n} b_{j, k} f_{k}\right\|_{\omega} \leq\left\|h_{j}\right\|_{\tilde{\omega}}+\sum_{k=1}^{n} \sum_{m=0}^{\infty}\left|\widehat{b_{j, k}} f_{k}(m)\right| \omega(m),
$$


où $\widehat{b_{j, k} f_{k}}(m)$ est le $m^{\text {ième }}$ coefficient de Fourier de la restriction de $b_{j, k} f_{k}$ au cercle unité. D'après (5), $\|F\|_{\widetilde{\omega}} \leq 1$ et $F(\zeta) \geq \delta^{2}$ pour tout $\zeta \in \mathrm{T}$. Donc

$$
\left\|h_{j}\right\|_{\widetilde{\omega}} \leq\left\|f_{j}\right\|_{\omega}\left\|F^{-1}\right\|_{\widetilde{\omega}} \leq\left\|f_{j}\right\|_{\omega} C_{1}\left(\delta^{2}, A_{\widetilde{\omega}}\right) .
$$

Notons aussi que

$$
\begin{aligned}
\widehat{b_{j, k}}(m) & =\frac{1}{2 \pi} \int_{0}^{2 \pi} b_{j, k}\left(e^{i t}\right) e^{-i m t} d t \\
& =-\frac{1}{\pi} \int_{\mathrm{D}} a_{j, k}(\zeta)\left(\frac{1}{2 \pi} \int_{0}^{2 \pi} \frac{e^{-i m t}}{\zeta-e^{i t}} d t\right) d A(\zeta) .
\end{aligned}
$$

Donc pour $m \geq 0, \widehat{b_{j, k}}(m)=0$ et pour $m \leq-1$, on a

$$
\begin{aligned}
\widehat{b_{j, k}}(m) & =\frac{1}{\pi} \int_{\mathrm{D}} a_{j, k}(\zeta) \zeta^{-m-1} d A(\zeta) \\
& =\frac{1}{\pi} \int_{0}^{1} \int_{0}^{2 \pi} a_{j, k}\left(r e^{i t}\right) r^{-m-1} e^{-i(m+1)} r d r d t \\
& =2 \int_{0}^{1} \widehat{a_{j, k}^{(r)}}(m+1) r^{-m} d r .
\end{aligned}
$$

Ici, lorsqu' une fonction $g$ est définie sur $\overline{\mathrm{D}}$, on note $g^{(r)}(z)=g(r z)$ pour $z \in \overline{\mathrm{D}}$ et $0 \leq r<1$. On obtient alors

$$
\begin{aligned}
\sum_{m=0}^{\infty}\left|\widehat{b_{j, k} f_{k}}(m)\right| \omega(m) & =\sum_{m=0}^{\infty}\left|\sum_{l=0}^{\infty} \widehat{b_{j, k}}(-l) \hat{f}_{k}(m+l) \omega(m)\right| \\
& \leq \sum_{l=0}^{\infty}\left|\widehat{b_{j, k}}(-l)\right| \sum_{m=0}^{\infty}\left|\hat{f}_{k}(m+l)\right| \omega(m+l) \\
& \leq\left\|f_{k}\right\|_{\omega} \sum_{l=0}^{\infty}\left|\widehat{b_{j, k}}(-l)\right| \\
& \leq 2\left\|f_{k}\right\|_{\omega} \sum_{l=0}^{\infty} \int_{0}^{1}\left|\widehat{a_{j, k}^{(r)}}(1-l)\right| r^{l} d r \\
& \leq 2\left\|f_{k}\right\|_{\omega} \int_{0}^{1}\left\|a_{j, k}^{(r)}\right\|_{1} d r .
\end{aligned}
$$

Des calculs bien connus montrent que $a_{j, k}=\left(\overline{f_{j}^{\prime}} \overline{f_{k}}-\overline{f_{j}} \overline{f_{k}^{\prime}}\right) F^{-2}$. Par con- 
séquent on a

$$
\begin{aligned}
\left\|a_{j, k}^{(r)}\right\|_{1} & =\left\|\frac{1}{F^{2(r)}}\left(\overline{f_{j}^{\prime(r)} f_{k}^{(r)}}-\overline{f_{j}^{(r)} f_{k}^{\prime(r)}}\right)\right\|_{1} \\
& \leq\left\|\frac{1}{F^{(r)}}\right\|_{1}^{2}\left(\left\|f_{j}^{\prime(r)}\right\|_{1}\left\|f_{k}^{(r)}\right\|_{1}+\left\|f_{j}^{(r)}\right\|_{1}\left\|f_{k}^{\prime(r)}\right\|_{1}\right) \\
& \leq C_{1}\left(\delta^{2}, A_{\widetilde{\omega}}\right)^{2}\left(\left\|f_{j}^{\prime(r)}\right\|_{1}\left\|f_{k}\right\|_{\omega}+\left\|f_{j}\right\|_{\omega}\left\|f_{k}^{((r)}\right\|_{1}\right) .
\end{aligned}
$$

Donc

$$
\int_{0}^{1}\left\|a_{j, k}^{(r)}\right\|_{1} d r \leq C_{1}\left(\delta^{2}, A_{\widetilde{\omega}}\right)^{2}\left(\left\|f_{k}\right\|_{\omega} \int_{0}^{1}\left\|f_{j}^{\prime(r)}\right\|_{1} d r+\left\|f_{j}\right\|_{\omega} \int_{0}^{1}\left\|f_{k}^{\prime(r)}\right\|_{1} d r\right) .
$$

Or pour tout $l=1,2, \ldots, n$, on a

$$
\int_{0}^{1}\left\|f_{l}^{\prime(r)}\right\|_{1} d r=\int_{0}^{1} \sum_{m \geq 1} m\left|\hat{f}_{l}(m)\right| r^{m-1} d r=\sum_{m \geq 1}\left|\hat{f}_{l}(m)\right| \leq\left\|f_{l}\right\|_{\omega} .
$$

Ce qui donne

$$
\int_{0}^{1}\left\|a_{j, k}^{(r)}\right\|_{1} d r \leq 2 C_{1}\left(\delta^{2}, A_{\widetilde{\omega}}\right)^{2}\left\|f_{j}\right\|_{\omega}\left\|f_{k}\right\|_{\omega}
$$

En combinant les inégalités (5), (6), (7), (8) et (9), on obtient

$$
\left\|g_{j}\right\|_{\omega} \leq\left\|f_{j}\right\|_{\omega} C_{1}\left(\delta^{2}, A_{\widetilde{\omega}}\right)+4\left\|f_{j}\right\|_{\omega} C_{1}\left(\delta^{2}, A_{\widetilde{\omega}}\right)^{2} \leq 5\left\|f_{j}\right\|_{\omega} C_{1}\left(\delta^{2}, A_{\widetilde{\omega}}\right)^{2} .
$$

D'où

$$
\left(\sum_{j=1}^{n}\left\|g_{j}\right\|_{\omega}^{2}\right)^{\frac{1}{2}} \leq 5 C_{1}\left(\delta^{2}, A_{\widetilde{\omega}}\right)^{2}
$$

Dans le cas où les $f_{j}$ ne sont pas holomorphes dans un voisinage de $\overline{\mathrm{D}}$ on considère pour $0<r<1$, les $f_{j}^{(r)}$ qui vérifient aussi la condition (5). D'après ce qui précède, il existe $g_{r}=\left(g_{r, 1}, \ldots, g_{r, n}\right) \in\left(A_{\omega}^{+}\right)^{n}$ satisfaisant

$$
\sum_{i=1}^{n} f_{i}^{(r)} g_{r, i}=1 \quad \text { et } \quad \sum_{i=1}^{n}\left\|g_{r, i}\right\|_{\omega}^{2} \leq 25 C_{1}\left(\delta^{2}, A_{\widetilde{\omega}}\right)^{4} .
$$

Comme $A_{\omega}^{+}$admet un prédual $\left(A_{\omega}^{+}\right.$peut être identifié au dual de $c_{0}(1 / \omega)=$ $\left.\left\{\left(u_{n}\right)_{n \geq 0}: \sup _{n \geq 0}\left|u_{n}\right| / \omega(n)<\infty\right\}\right)$, d'après le théorème de Banach-Alaoglu, la boule unité fermée $B_{\omega}$ de $A_{\omega}^{+}$est compacte pour la topologie faible-*. 
Comme de plus $c_{0}(1 / \omega)$ est séparable, $B_{\omega}$ est metrisable pour la topologie faible-*. Il existe alors une suite croissante $\left(r_{k}\right)$ tendant vers 1 et $g=$ $\left(g_{1}, \ldots, g_{n}\right) \in\left(A_{\omega}^{+}\right)^{n}$ tel que pour $1 \leq i \leq n,\left(g_{r_{k}, i}\right)_{k}$ converge faiblement vers $g_{i}$. Comme la convergence faible entraine la convergence uniforme sur tout compact de $\mathrm{D}$, on obtient

$$
\sum_{i=1}^{n} f_{i} g_{i}=1 \quad \text { et } \quad \sum_{i=1}^{n}\left\|g_{i}\right\|_{\omega}^{2} \leq 25 C_{1}\left(\delta^{2}, A_{\widetilde{\omega}}\right)^{4} .
$$

\section{Preuve du Théorème $B$}

Si $f \in A(\mathrm{D})$, on notera $Z(f)=\{z \in \overline{\mathrm{D}}: f(z)=0\}$ et si $M \subset A(\mathrm{D})$, on notera $Z(M)=\cap_{f \in M} Z(f)$. Pour tout fermé $E \subset \overline{\mathrm{D}}$ on posera $E_{\delta}=\{\zeta \in \overline{\mathrm{D}}$ : $d(\zeta, E) \leq \delta\}$, où $d(\zeta, E)$ désigne la distance de $\zeta$ à $E$.

Pour la preuve du résultat principal de cette section nous aurons besoin des deux lemmes élémentaires suivants.

Lemme 3.1. Soit I un idéal fermé de $A_{\omega}^{+}$où $\omega$ est un poids satisfaisant (3) et soit $E=Z(I)$. Pour tout $\delta>0$, il existe $f \in I$ tel que pour tout $z \in \overline{\mathrm{D}} \backslash E_{\delta}$, $f(z) \neq 0$.

Preuve. Soit $I$ un idéal fermé de $A_{\omega}^{+}$tel que $Z(I)=E$. La ferméture $J$ de $I$ dans $A(\mathrm{D})$ est un idéal fermé tel que $Z(J)=E$. D'après la caractérisation des idéaux fermés de $A(D)$ ([6]), il existe $g \in J$ tel que $Z(g)=E$. En approchant $g$ par les éléments de $I$ on obtient le résultat.

On note alors

$$
\varrho_{I}(\delta)=\sup \left\{\inf \left\{|f(z)|: z \in \overline{\mathrm{D}} \backslash E_{\delta}\right\}\right\},
$$

le sup étant pris sur les $f \in I$ tels que $\|f\|_{\omega} \leq 1$. La conclusion du lemme 3.1 se traduit alors $\operatorname{par} \varrho_{I}(\delta)>0$ pour tout $\delta>0$.

Pour toute suite $(\omega(n))_{n \geq 0}$, on notera par $\omega_{*}$ la fonction réciproque définie sur $] 1,+\infty[$ par

$$
\omega_{*}(t)=\inf \{n \geq 1: \omega(n) \geq t\},
$$

avec la convention inf $\emptyset=+\infty$.

Lemme 3.2. Soit $\omega$ une suite croissante satisfaisant (3), alors pour tout $z, \zeta \in \overline{\mathrm{D}}$ tels que $|z-\zeta| \leq \frac{\delta}{\omega_{*}(2 / \delta)}$ on $a|f(z)-f(\zeta)| \leq \delta\|f\|_{\omega}$ pour tout $f \in A_{\omega}^{+}$. 
Preuve. Soit $f \in A_{\omega}^{+}$et soit $z, \zeta \in \overline{\mathrm{D}}$ on a:

$$
|f(z)-f(\zeta)| \leq \sum_{n \geq 1}\left|\hat{f}(n)\left\|z^{n}-\zeta^{n} \mid \leq \sup _{n \geq 1} \frac{\left|z^{n}-\zeta^{n}\right|}{\omega(n)}\right\| f \|_{\omega}\right.
$$

D'une part si $n \geq \omega_{*}(2 / \delta)$ alors $\frac{\left|z^{n}-\zeta^{n}\right|}{\omega(n)} \leq \frac{2}{\omega\left(\omega_{*}(2 / \delta)\right)} \leq \delta$. D'autre part si $n \leq \omega_{*}(2 / \delta)$ alors $\frac{\left|z^{n}-\zeta^{n}\right|}{\omega(n)} \leq|z-\zeta| n \leq|z-\zeta| \omega_{*}(2 / \delta) \leq \delta$ dès que $|z-\zeta| \leq$ $\frac{\delta}{\omega_{*}(2 / \delta)}$. On a donc bien $\sup _{n \geq 1} \frac{\left|z^{n}-\zeta^{n}\right|}{\omega(n)} \leq \delta$ pour $|z-\zeta| \leq \frac{\delta}{\omega_{*}(2 / \delta)}$.

Pour démontrer l'impliction ii) $\Rightarrow$ i) dans le théorème $B$, il suffit de prendre $I=\{0\}$ et d'utiliser ensuite le Théorème A. Quand à l'implication i) $\Rightarrow$ ii) elle découle du résultat suivant. On rappelle que pour les suites $\omega$ croissantes, la condition i) dans le théorème $\mathrm{B}$ entraine que $\omega$ diverge vers l'infini, ce qui assure que $\omega_{*}(t)$ est fini pour tout $t$.

THÉORÈME 3.3. Soit $\omega$ une suite croissante satisfaisant (3) et soit I un idéal fermé de $A_{\omega}^{+}$alors

$$
C_{n}\left(\delta, A_{\omega}^{+} / I\right) \leq \frac{5}{\sqrt{2}} C_{1}\left(M_{I}(\delta)^{2}, A_{\widetilde{\omega}}\right)^{2},
$$

où $M_{I}(\delta)=\operatorname{Min}\left(\frac{1}{4} \varrho_{I}\left(\frac{\delta^{2}}{4 \omega_{*}\left(8 / \delta^{2}\right)}\right), \frac{\delta}{2 \sqrt{2}}\right)$

Preuve. Soit $\delta \in] 0,1]$ et soit $f=\left(f_{1}, f_{2}, \ldots, f_{n}\right) \in\left(A_{\omega}^{+}\right)^{n}$ satisfaisant

$$
\sum_{j=1}^{n}\left\|f_{j}\right\|_{\omega}^{2} \leq 3 / 2, \quad \sum_{j=1}^{n}\left|f_{j}(\zeta)\right|^{2} \geq \delta^{2} \quad \text { pour tout } \zeta \in E:=Z(I) .
$$

Soit $\delta^{\prime}=\frac{\delta^{2}}{4 \omega_{*}\left(8 / \delta^{2}\right)}$. D'après le lemme 3.2, si $|z-\zeta| \leq \delta^{\prime}$, alors $\left|f_{j}(z)-f_{j}(\zeta)\right| \leq$ $\left(\delta^{2} / 4\right)\left\|f_{j}\right\|_{\omega}$. Donc pour tout $\zeta \in E_{\delta^{\prime}}$ il existe $z \in E$ tel que $|\zeta-z| \leq \delta^{\prime}$ et par conséquent

$$
\begin{aligned}
\sum_{j=1}^{n}\left|f_{j}(\zeta)\right|^{2} & =\sum_{j=1}^{n}\left|f_{j}(z)\right|^{2}-\sum_{j=1}^{n}\left(\left|f_{j}(z)\right|^{2}-\left|f_{j}(\zeta)\right|^{2}\right) \\
& \geq \delta^{2}-\sum_{j=1}^{n}\left|f_{j}(z)-f_{j}(\zeta)\right|\left|f_{j}(z)+f_{j}(\zeta)\right| \\
& \geq \delta^{2}-\sum_{j=1}^{n} \frac{\delta^{2}}{4}\left(2\left\|f_{j}\right\|_{\omega}^{2}\right) \\
& \geq \delta^{2} / 4 .
\end{aligned}
$$


D'après le lemme 3.1, il existe $h \in I$ tel que $\|h\|_{\omega}=1$ et $|h(z)| \geq \varrho_{I}\left(\delta^{\prime}\right) / 2$ pour tout $z \in \mathrm{T} \backslash E_{\delta^{\prime}}$. On pose alors pour $j=1,2, \ldots, n, h_{j}=\frac{1}{\sqrt{2}} f_{j}$ et $h_{n+1}=$ $\frac{1}{2} h$. Il est alors clair que $\sum_{j=1}^{n+1}\left\|h_{j}\right\|_{\omega}^{2} \leq 1$ et que $\sum_{j=1}^{n+1}\left|h_{j}(z)\right|^{2} \geq M_{I}(\delta)^{2}$ pour tout $z \in \overline{\mathrm{D}}$. D' après le théorème 2.1 , il existe $g_{1}, g_{2}, \ldots, g_{n+1} \in A_{\omega}^{+}$tels que $\sum_{j=1}^{n+1} h_{j} g_{j}=1$ et $\sum_{j=1}^{n+1}\left\|g_{j}\right\|^{2} \leq 25 C_{1}\left(M_{I}(\delta)^{2}, A_{\widetilde{\omega}}\right)^{4}$. Finalement pour $k_{j}=(1 / \sqrt{2}) g_{j}, j=1,2, \ldots, n$, on obtient

$$
\sum_{j=1}^{n} \pi\left(f_{j}\right) \pi\left(k_{j}\right)=\pi(1) \quad \text { et } \quad \sum_{j=1}^{n}\left\|k_{j}\right\|^{2} \leq(25 / 2) C_{1}\left(M_{I}(\delta)^{2}, A_{\widetilde{\omega}}\right)^{4},
$$

où $\pi$ est la surjection canonique de $A_{\omega}^{+}$dans $A_{\omega}^{+} / I$, ce qui achève la preuve.

Remarque. Soit $p \geq 1$ et $\omega=\left(\omega_{n}\right)_{n \geq 0}$ une suite croissante telle que $\omega(0)=1$ et $\lim _{n \rightarrow+\infty} \omega(n)^{\frac{1}{n}}=1$. On pose

$$
A_{\omega, p}^{+}=\left\{f \in A(\mathrm{D}):\|f\|_{\omega, p}=\left(\sum_{n \in \mathrm{N}}|\hat{f}(n)|^{p} \omega(n)^{p}\right)^{\frac{1}{p}}<+\infty\right\} .
$$

Si on suppose que $A_{\omega, p}^{+}$est une algèbre topologique i.e il existe une constante $C>0$ telle que

$$
\|f g\|_{\omega, p} \leq C\|f\|_{\omega, p}\|g\|_{\omega, p},\left(f, g \in A_{\omega, p}^{+}\right),
$$

alors les conclusions des théorèmes $\mathrm{A}$ et $\mathrm{B}$ sont vraies pour $A_{\omega, p}^{+}$.

\section{Exemples et Remarques}

Soit $A$ une algèbre de Banach commutative unitaire et soit $I$ est un idéal fermé de $A$. L'ensemble des caractères de l'algèbre quotient $A / I$ s'identifie à l'ensemble $Z(I)=\{\chi \in \hat{A}: \hat{f}(\chi)=0, \chi \in I\}$ ([3]). On a le résultat suivant.

ThÉORÈme 4.1. Supposons que la transformée de Gelfand de A soit compacte. Alors pour tout $\delta \in(0,1]$, il existe une constante $M(\delta) \in(0,1]$ telle que

i) Pour tout entier $n \geq 1$, il existe un entier $m \geq 1$ tel que

$$
C_{n}(\delta, A / I) \leq C_{m}(M(\delta), A) .
$$

En particulier si le spectre de A est $\delta$-complètement visible pour tout $\delta \in(0,1]$, alors il en est de même pour le spectre de A/I.

ii) Si de plus I satisfait la condition suivante: Pour tout voisinage $V$ de $Z(I)$,

$$
\text { il existe } f \in I \text { tel que } \hat{f}(\chi) \neq 0 \text { pour tout } \chi \notin V \text {, }
$$


alors

$$
C_{n}(\delta, A / I) \leq C_{n+1}(M(\delta), A)
$$

En particulier $\delta_{n+1}(A)=0 \Longrightarrow \delta_{n}(A / I)=0$.

La preuve de ce théorème utilise le théorème de Arzèla-Ascoli et des arguments similaires à ceux utilisés dans la preuve du théorème 3.3. Nous l'omittons ici.

On dira que $A$ est symétrique s'il existe une constante $c>0$ telle que pour tout $f \in A$ on peut trouver $f^{\star} \in A$ tel que $\left\|f^{\star}\right\| \leq c\|f\|$ et $\hat{f}^{\star}(\chi)=\overline{\hat{f}(\chi)}$, $\chi \in \hat{A}$. Si $A$ est symétrique et si $\left(f_{1}, \ldots, f_{n}\right) \in A^{n}$ satisfait la condition (1) alors on a $\sum_{i=1}^{n} f_{i} g_{i}=e$, où $g_{i}=f_{i}^{\star}\left(\sum_{i=1}^{n} f_{i} f_{i}^{\star}\right)^{-1}, 1 \leq i \leq n$. Ceci montre que $C_{n}(\delta, A) \leq C_{1}\left(\delta^{2} / c, A\right), n \geq 1$. Ainsi si le spectre de $A$ est $\left(\frac{\delta^{2}}{c}-1\right)$-visible alors il est $\delta$-complètement visible (voir aussi [9]).

D'autre part il est facile de voir que tout idéal fermé d'une algèbre symétrique satisfait la condition (10). Il découle alors de ces observations et du théorème 4.1 que si $A$ est symétrique et si sa transformée de Gelfand est compacte alors

$$
\delta_{n+1}(A)=0 \Longrightarrow \delta_{n}(A / I)=0 .
$$

Considérons maintenant une algèbre $A$ telle que $\mathscr{G}(A)$, l'image de $A$ par sa transformée de Gelfand $\mathscr{G}$, est dense dans $\mathscr{C}(\hat{A})$, pour la norme de la convergence uniforme sur $\hat{A}$. Nous clamons que si $I$ est un idéal fermé de $A$ alors il satisfait la condition (10). En effet, soit $J$ la fermeture de $\mathscr{G}(I)$ dans $\mathscr{C}(\hat{A})$, alors $J$ est un idéal fermé et $Z(J)=Z(I)$. Soit $V$ un voisinage de $Z(I)$ et soit $\varphi \in \mathscr{C}(\hat{A})$ une fonction égale à 1 sur $Z(I)$ et dont le support est contenu dans $V$. Alors d'après la structure des idéaux fermés de $\mathscr{C}(\hat{A})$ (voir [2], Theorem 4.1.3), la fonction $1-\varphi$ est dans $J$ et en l'approchant par des fonctions de $\mathscr{G}(I)$ on voit que $I$ satisfait la condition (10).

Enfin nous signalons que la condition (10) n'est pas toujours satisfaite comme le montre l'exemple suivant :

Soit $A\left(\mathrm{D}^{2}\right)=\left\{f \in \mathscr{C}\left(\overline{\mathrm{D}^{2}}\right): f\right.$ holomorphe sur $\left.\mathrm{D}^{2}\right\}$, l'algèbre du bidisque. Muni du produit ponctuel et de la norme $\|f\|_{\infty}=\sup _{z \in \mathrm{D}^{2}}|f(z)|, A\left(\mathrm{D}^{2}\right)$ est une algèbre de Banach commutative et unitaire. Son spectre peut être identifié à $\overline{\mathrm{D}^{2}}$. Soit $K \subset \mathrm{D}^{2}$ un compact et soit $I(K)$ l'idéal fermé de $A\left(\mathrm{D}^{2}\right)$ définit par $I(K)=\left\{f \in A\left(\mathrm{D}^{2}\right): f_{\mid K} \equiv 0\right\}$. On peut vérifier aisément, grâce au théorème de Hartogs, que $I(K)$ ne vérifie pas (10).

Dans la suite nous allons considérer deux exemples d'algèbres de Banach dans les transformées de Gelfand ne sont pas compactes.

1) Rappelons qu'on note par $A(\mathrm{D})$ l'algèbre du disque. Il est bien clair que $\delta_{1}(A(\mathrm{D}))=0$ et que pour tout $\delta \in(0,1], C_{1}(\delta, A(\mathrm{D}))=1 / \delta$. La proposition 
suivante montre, en particulier, qu'il existe des idéaux fermés $I$ de $A(D)$ tel que $\delta_{1}(A(\mathrm{D}) / I)=1$.

Proposition 4.2. Soit I un idéal fermé de $A(\mathrm{D})$ tel que $Z(I) \subset \mathrm{T}$.

(i) Si $I=\{f \in A(\mathrm{D}): f(z)=0(z \in Z(I))\}$, alors pour tout $n \geq 1$, $\delta_{n}(A(\mathrm{D}) / I)=0$.

(ii) Si $I \neq\{f \in A(\mathrm{D}): f(z)=0(z \in Z(I))\}$, alors pour tout $n \geq 1$, $\delta_{n}(A(\mathrm{D}) / I)=1$.

Preuve. Posons $E=Z(I)$ et supposons que $I=\{f \in A(\mathrm{D}): f(z)=$ $0(z \in E)\}$. Notons que $E$ est de mesure de Lebesgue nulle. D'après le théorème de Rudin-Bishop, toute fonction continue sur $E$ est la restiction à $E$ d'une fonction dans $A(\mathrm{D})$ ([6]). En fait l'injection $f+I \rightarrow f_{\mid E}$ est une isométrie bijective de $A(\mathrm{D}) / I$ dans $\mathscr{C}(E)$. On a alors $C_{n}(\delta, A(\mathrm{D}) / I)=$ $C_{n}(\delta, \mathscr{C}(E))=1 / \delta, n \geq 1,0 \leq \delta<1$. Ainsi la partie i) de la proposition est bien vérifiée.

Soient $\pi$ la surjection canonique de $A(\mathrm{D})$ dans $A(\mathrm{D}) / I, u: z \rightarrow z$ la fonction identité et $f_{n}=u^{n}, n \geq 1$. Supposons qu' il existe une constante $C>0$ telle que $\left\|\pi\left(f_{n}\right)^{-1}\right\|_{\infty} \leq C, n \geq 1$. Pour tout polynôme trignômétrique $p\left(e^{i t}\right)=\sum_{n=-N}^{N} a_{n} e^{i n t}$, on a

$$
\begin{aligned}
\|p(\pi(u))\|_{\infty} & =\left\|\pi(u)^{-N} \sum_{n=-N}^{N} a_{n} \pi(u)^{n+N}\right\|_{\infty} \\
& \leq\left\|\pi(u)^{-N}\right\|_{\infty}\left\|\sum_{n=-N}^{N} a_{n} \pi(u)^{n+N}\right\|_{\infty} \\
& \leq C\left\|\sum_{n=-N}^{N} a_{n} u^{n+N}\right\|_{\infty} \\
& \leq C\|p\|_{\infty} .
\end{aligned}
$$

Donc il existe un morphisme continu $\Phi$ de $\mathscr{C}(\mathrm{T})$ dans $A$ (D)/I prolongeant $\pi$. Le noyau Ker $\Phi$ est un ideal fermé de $\mathscr{C}(\mathrm{T})$ qui verifie $\operatorname{Ker} \Phi \cap A(\mathrm{D})=I$. Donc $\{f \in \mathscr{C}(\mathrm{T}): f(z)=0(z \in E)\} \subset \operatorname{Ker} \Phi$. D'où $I=\{f \in A(\mathrm{D})$ : $f(z)=0(z \in E)\}$.

Supposons maintenant que $I \neq\{f \in A(\mathrm{D}): f(z)=0(z \in E)\}$. D'après ce qui précède on a $\sup _{n}\left\|f_{n}\right\|_{\infty}=+\infty$. Puisque pour tout $n,\left\|f_{n}\right\|_{\infty}=1$ et $\left|f_{n}(z)\right|=1,(z \in E)$, on obtient que $\delta_{1}(A(\mathrm{D}) / I)=1$. Comme $\delta_{n}(A(\mathrm{D}) / I) \geq$ $\delta_{1}(A(\mathrm{D}) / I), n \geq 1$, on voit que la partie ii) de la proposition est satisfaite. 
2) Considérons maintenant l'algèbre de Wiener analytique

$$
A^{+}=\left\{f \in A(\mathrm{D}):\|f\|_{1}=\sum_{n \geq 0}|\hat{f}(n)|<+\infty\right\} .
$$

Il est montré dans [5] (voir aussi [9]) que $\delta_{1}\left(A^{+}\right)=\frac{1}{2}, C_{1}\left(\delta, A^{+}\right)=\frac{1}{1-2 \delta}$ pour $\frac{1}{2}<\delta \leq 1$ et $C_{1}\left(\delta, A^{+}\right)=+\infty$ pour $0<\delta \leq \frac{1}{2}$.

Pour un fermé non vide $E$ de T, on pose $I_{E}=\left\{f \in A^{+}: f(z)=0, \quad(z \in\right.$ $E)\}$. On supposera que $E$ est de type $Z A^{+}$i.e $I_{E} \neq\{0\}$. Contrairement à ce qui se passe dans la proposition 4.2, les constantes $\delta_{n}\left(A^{+} / I_{E}\right)$ dépendent de $E$. En effet si $E$ est un ensemble de Helson (voir [7] pour la définition) alors d'après le résultat de Wik [12], on a $A^{+}(E)=C(E)$, où $A^{+}(E)$ désigne l'algèbre des restrictions des fonctions de $A^{+}$à $E$. Dans ce cas on a $\delta_{n}\left(A^{+} / I_{E}\right)=0, n \geq 1$. D'autre part si $E$ n'est pas de type $A A^{+}$(voir [8]) alors on a $\left\|\pi\left(u^{n}\right)\right\|=1$ pour $n \geq 1,\left|u^{n}(z)\right|=1$ pour $z \in E$ et $\lim _{n \rightarrow+\infty}\left\|\pi(u)^{-n}\right\|=+\infty$, où $\pi$ est la surjection canonique de $A^{+}$dans $A^{+} / I_{E}$ et $u$ la fonction identité $z \rightarrow z$. On voit alors que dans ce cas on a $\delta_{1}\left(A^{+} / I_{E}\right)=1$ et par conséquent $\delta_{n}\left(A^{+} / I_{E}\right)=1$, $n \geq 1$.

\section{REFERENCES}

1. Aleman, A., and Dahlner, A., Uniform spectral radius and compact gelfand transforms, Préprint.

2. Aupetit, B., A Primer on Spectral Theory, Springer Verlag, New York, 1991.

3. Bonsal, F. F., and Duncan, J., Complete Normed Algebras, Springer Verlag, New York, 1958.

4. El-Fallah, O., and Ezzaaraoui, A., Majorations uniformes de normes d'inverses dans les Algèbres de Beurling, J. London Math. Soc. (2) 65 (2002), 705-719.

5. El-Fallah, O., Nikolskii, N. K., and Zarrabi, M., Resolvant estimates in Beurling-Sobolev algebras, St. Petersburg Math. J. 10, no 6 (1999), 901-964.

6. Hoffman, K., Banach Spaces of Analytic Functions, Prentice Hall, Englewood Cliffs, NewJersey, 1962.

7. Kahane, J. P., Séries de Fourier absolument convergentes, Springer Verlag, Berlin-Heidelberg-New York, 1970.

8. Kahane, J. P., Katznelson, Y., Sur les algèbres de restrictions des séries de Taylor absolument convergentes à un fermé du cercle, J. Analyse Math. 23 (1970), 185-197.

9. Nikolskii, N. K., In search of the invisible spectrum, Ann. Inst. Fourier (Grenoble) 49 (1999), 1925-1998.

10. Nikolskii, N. K., The phenomenon of the invisible spectrum and the problem of efficient inversions, Proc. Conference "Harmonic Analysis in 20th century", ed. J. Byrnes.

11. Tolokonnikov, V. A., The corona theorem in algebras of bounded analytic functions, Amer. Math. Soc. Transl. Ser. 2149 (1991), 61-95. 
12. Wik, I., On linear dependence in closed sets, Ark. Mat. 4 (1961), 209-218.

\author{
UNIVERITÉ MOHAMED V \\ DÉPARTEMENT DE MATHÉMATIQUES ET INFORMATIQUE \\ AVENUE IBN BATTOUTA \\ BP 1014, RABAT \\ MAROC \\ E-mail: elfallah@fsr.ac.ma
}

\author{
UNIVERSITÉ BORDEAUX I \\ LABORATOIRE DE MATHÉMATIQUES PURES \\ UMR 5467 \\ 351, COURS DE LA LIBÉRATION \\ 33405 TALENCE CEDEX \\ FRANCE \\ E-mail: zarrabi@math.u-bordeaux.fr
}

